\title{
Neurological Disease Burden: Bangladesh Perspective
}

\author{
Md. Badrul Alam \\ Professor of Neurology \& Joint Director, National Institute of Neurosciences \& Hospital, Sher-E-Bangla Nagar, \\ Agargaon, Dhaka, Bangladesh; Email:badrul_dmc@yahoo.com
}

Neurologic disorders represent a major burden of disease globally. These are common and represent a major public health problem. The spectrum of diseases ranges from non-communicable disorders like stroke and neurodegenerative disorders to central nervous system infections. The burden of neurological diseases may be on the increase especially in developing countries ${ }^{1}$. Improved outcome in these settings may require appreciation of the spectrum of Neurological diseases and the impediments to their management.

The Global Burden of Disease (GBD) study which is a collaborative endeavour of the World Health Organization (WHO), the World Bank and the Harvard School of Public Health, drew the attention of the international health community to the burden of neurological disorders and many other chronic conditions ${ }^{2}$. The burden of neurological disorders is seriously underestimated by traditional epidemiological and health statistical methods that take into account only mortality rates but not disability rates ${ }^{2}$. The GBD study showed that over the years the global health impact of neurological disorders had been underestimated ${ }^{3}$. World health organization (WHO) has estimated that 50 million people have epilepsy ${ }^{2}$. It is projected that the number of people affected by dementia will double every 20 years $^{2}$. Neurological disorders and some of the other conditions with neurological impairments and sequelae constitute over $6 \%$ of the global burden of disease and this burden is especially high in many low and middle income countries ${ }^{3}$.

The pattern of neurological admissions in hospital varies amongst different regions of the world and this depends on many factors including the regional burden of neurological disorders. Philip-Ephraim et $\mathrm{al}^{4}$ have reported that neurological diseases is constituted $24.2 \%$ of all medical conditions seen over a one year period and stroke is found to be the commonest cause of admissions accounting for $42.1 \%$ of the cases followed by peripheral neuropathy $(13.8 \%)$ and meningoencephalitis (7.2\%). Thus it is very obvious that stroke remains the most frequent cause of neurologic admissions and mortality. Eze and Kalu ${ }^{5}$ have reported that stroke accounted for $62 \%$ of the neurologic admissions. Others were central nervous system (CNS) infections, seizure disorders, hypertensive encephalopathy, myelopathies, CNS tumors and neurodegenerative disorders in descending order of frequency. Stroke and CNS infections were the most prevalent neurological disorders identified. In another study Ekenze et al $^{1}$ have found that neurological admissions comprise about $14.8 \%$ of medical admissions; furthermore, the spectrum of neurological diseases are stroke $64.9 \%$, central nervous system infections (21.8\%), HIV related neurological diseases (3.5\%), hypertensive encephalopathy (3.4\%), dementia (3\%), subarachnoid haemorrhage (2.2\%), Guillian Barre syndrome $(1.2 \%)$, Parkinson's disease (1.1\%), myasthenia gravis $(1.0 \%)$, motor neuron disease and peripheral neuropathy and accounted for $0.8 \%$ and $0.6 \%$ respectively. Overall, noninfectious disease accounted for $78.2 \%$ of neurological admissions while infectious diseases accounted for $11.8 \% \%^{5}$. A wide spectrum of neurological diseases occurs. The high incidence of CNS infections indicates that efforts should be geared towards preventive measures. A major challenge to be addressed in the management of neurological diseases is the lack of specialized facilities ${ }^{2}$.

Neurology is introduced as a specialty in Bangladesh ${ }^{6}$ during the 1960s'. An excess burden of cerebrovascular disease and stroke denotes a higher risk of mortality and morbidity in Bangladesh ${ }^{7}$. As neurologic disorders are quite common among all medical admissions in tertiary care hospital in Bangladesh and there are lack of facilities elsewhere in the country, varieties of neurological problems are referred to tertiary referral neurological institute named National Institute of Neurosciences and Hospital (NINS\&H).

Though the number of neurologists has increased over 
last decade in Bangladesh, it is still not enough ${ }^{6}$. Many patients with neurological problems are often dealt by internists and others from different specialties. Studies have proved the usefulness of liaison with neurology, especially in referral tertiary care neurology hospital like NINS\&H and the patient care also improved with specialist management. A comprehensive survey of inpatient admission of neurological problems and referral seeking behavior of different departments should be carried out. It gives a transparent idea about the burden of neurological cases in these departments. Stroke and CNS infectionsare both largely preventable. There should be good health planning that will address the enormous neurological disease burden with emphasis on preventive health.

[Journal of National Institute of Neurosciences Bangladesh 2015; 1(2): 31-32]

\section{References}

1. Ekenze OS1, Onwuekwe IO, Ezeala Adikaibe BA. Profile of neurological admissions at the University of Nigeria Teaching Hospital Enugu. Niger J Med. 2010;19(4):419-22

2. World Health Organization. Neurological disorders: public health challenges. World Health Organization; 2006.; Viewed on: 9 September 2016; Web Address: http://www.who.int/ mental_health/ neurology/neurodiso/en/

3. Murray CJL, Lopez AD, eds. The global burden of disease: a comprehensive assessment of mortality and disability from diseases, injuries and risk factors in 1990 and projected to 2020. Cambridge, MA, Harvard School of Public Health on behalf of the World Health Organization and the World Bank, 1996 (Global Burden of Disease and Injury Series, Vol. I)

4. Philip-Ephraim EE1, Eyong KI, Chinenye S, William UE, Ephraim RP. The burden of inpatient neurologic disease in a tropical African hospital. Can J Neurol Sci. 2013 Jul;40(4):576-9

5. Eze CO, Kalu UA. Pattern of neurological admissions in the tropics: experience at Abakaliki South-Eastern Nigeria. Niger J Med 2014;23(4):302-5

6. Chowdhury RN, Hasan ATMH, Rahman YU, Khan SI, Hussain AR, Ahsan S. Pattern of neurological disease seen among patients admitted in tertiary care hospital. BMC Research Notes 2014;7:202

7. Bhopal R, Rahemtulla T, Sheikh A: Persistent high stroke mortality in Bangladeshi populations. BMJ. 2005, 331: 1096-1097 